\title{
Philosophiques
}

\section{Le rasoir de Kant}

\section{Ruwen Ogien}

Volume 28, numéro 1, printemps 2001

\section{La nature des normes}

URI : https://id.erudit.org/iderudit/004963ar

DOI : https://doi.org/10.7202/004963ar

Aller au sommaire du numéro

\section{Éditeur(s)}

Société de philosophie du Québec

ISSN

0316-2923 (imprimé)

1492-1391 (numérique)

Découvrir la revue

\section{Citer cet article}

Ogien, R. (2001). Le rasoir de Kant. Philosophiques, 28(1), 9-25.

https://doi.org/10.7202/004963ar

\section{Résumé de l'article}

Dans cet article, je m'intéresse à quelques problèmes liés à la justification de méta-normes telles que " devoir " implique " pouvoir ". Je soutiens, d'abord, que des méta-normes différentes sont justifiées par des principes différents. La méta-norme " devoir » implique " pouvoir » est justifiée par un principe d'humanité qui nous demande d'exclure les normes trop exigeantes. La métanorme selon laquelle ce serait une sottise d'ordonner à quelqu'un ce qu'il veut inévitablement de lui-même ( « le rasoir de Kant ») est justifiée par un principe de parcimonie qui nous demande d'exclure les normes trop peu exigeantes. Je soutiens ensuite que ces deux principes (humanité et parcimonie) peuvent entrer en conflit. Ce qui m'amène à la conclusion que toute théorie normative qui s'efforce de respecter ces deux principes risque d'être instable.
Ce document est protégé par la loi sur le droit d'auteur. L'utilisation des services d'Érudit (y compris la reproduction) est assujettie à sa politique d'utilisation que vous pouvez consulter en ligne.

https://apropos.erudit.org/fr/usagers/politique-dutilisation/ 


\title{
Le rasoir de Kant
}

\author{
RUWEN OGIEN \\ CNRS \\ Ruwen@wanadoo.fr
}

\begin{abstract}
RÉSUMÉ. - Dans cet article, je m'intéresse à quelques problèmes liés à la justification de méta-normes telles que « devoir » implique « pouvoir ». Je soutiens, d'abord, que des méta-normes différentes sont justifiées par des principes différents. La méta-norme « devoir » implique « pouvoir » est justifiée par un principe d'humanité qui nous demande d'exclure les normes trop exigeantes. La métanorme selon laquelle ce serait une sottise d'ordonner à quelqu'un ce qu'il veut inévitablement de lui-même ( «le rasoir de Kant ») est justifiée par un principe de parcimonie qui nous demande d'exclure les normes trop peu exigeantes. Je soutiens ensuite que ces deux principes (humanité et parcimonie) peuvent entrer en conflit. Ce qui m'amène à la conclusion que toute théorie normative qui s'efforce de respecter ces deux principes risque d'être instable.
\end{abstract}

ABSTRACT. - The present paper adresses some problems related to the justification of meta-norms such as "ought" implies "can". First, I argue that different meta-norms are justified by different principles. The meta-norm "ought" implies "can" is justified by a principle of humanity ruling out norms that are too demanding. The meta-norm according to which it would be foolish to command of someone what he unavoidably wants already ("Kant's Razor") is justified by a principle of parsimony ruling out norms that are not demanding enough. I argue, then, that these two principles (humanity and parsimony) may conflict. This leads me to the conclusion that any normative theory trying to respect both principles may be unstable.

Certains philosophes considèrent que le principe ou, plus exactement, la méta-norme " devoir implique pouvoir » est un bon critère de sélection des théories morales ${ }^{1}$. D'après eux, une théorie morale est incohérente si elle ne respecte pas cette méta-norme dans ses prémisses et dans l'ensemble des propositions normatives qui en sont dérivées ${ }^{2}$. Pourtant, cette méta-norme est loin d'être totalement lumineuse, son interprétation correcte étant une affaire assez compliquée 3 . Parmi les questions qu'on se pose, à son propos, l'une des plus courantes est la suivante : qu'est-ce qui donne à cette métanorme son caractère apparemment inattaquable ? Est-ce l'existence d'une relation conceptuelle entre les termes « devoir » et « pouvoir » ?

1. J'ai choisi "méta-norme " pour des raisons que je propose dès la première section. J'ai suivi sur ce point Tranøy, K.E., " "Ought" implies "Can". A Bridge from Fact to Norm » Ratio,14, 1972, p. 116-130.

2. Brown, James, "Moral Theory and the Ought-Can Principle ", Mind, Avril 1977, p.206-223.

3. Williams, Bernard, "La cohérence éthique ", dans La fortune morale, trad.J. Lelaidier, Paris, PUF, 1994, p.118. Railton, Peter, "Made in the Shade : Moral Compatibilism and the Aims of Moral Theory ", Canadian Journal of Philosophy, Vol. suppl. 21, 1995, p. 91.

PHILOSOPHIQUES 28/1 — Printemps 2001, p. 9-25 
Dans ce qui suit, j'essaie, d'abord, de montrer que le caractère apparemment inattaquable de la méta-norme " devoir implique pouvoir » ne provient pas de l'existence d'une relation conceptuelle entre " devoir " et " pouvoir ». Ce qui lui donne ce caractère, à mon avis, c'est qu'elle est fondée sur un principe moral auquel nous ne sommes pas prêts à renoncer, un principe que j'appelle d' " humanité ", lequel nous recommande de ne pas formuler des normes exigeantes au point d'être cruelles ou inhumaines.

J'essaie, ensuite, d'établir que " devoir implique pouvoir » n'est pas la seule méta-norme qui puisse servir de critère de sélection des théories morales. Il en existe au moins une autre qui, me semble-t-il, n'a pas reçu le degré d'attention qu'elle méritait. Il est permis de dire que c'est à elle que Kant fait référence lorsqu'il écrit : "Un commandement ordonnant à chacun de chercher à se rendre heureux serait une sottise ; car on n'ordonne jamais à quelqu'un ce qu'il veut déjà inévitablement de lui-même ${ }^{4}$. Cette métanorme semble exclure la possibilité de normes qui nous obligeraient à chercher à obtenir ce que nous voulons de toute façon et de normes dérivées qui nous interdiraient de chercher à obtenir ce que nous ne voulons en aucun cas. À la différence de "devoir implique pouvoir ", cette méta-norme n'est pas justifiée par un principe d'humanité, mais par un principe de parcimonie, lequel nous demande d'éliminer les normes superflues.

On a pris l'habitude d'appeler « rasoir d'Occam » le principe de parcimonie ontologique qui nous recommande d'éliminer les entités superflues. Dans le même esprit, j'appellerai " rasoir de Kant », le principe de parcimonie qui nous recommande d'éliminer les normes superflues, puisque c'est en référence à une citation de Kant que j'ai suggéré qu'il existait un principe de ce genre.

Généralement, c'est plutôt la méta-norme "devoir implique pouvoir » qu'on attribue à Kant ${ }^{5}$. Cependant, la version de cette méta-norme qui m'intéresse est celle qui correspond à l'adage «A l'impossible nul n'est tenu ". Or, il y a d'assez bonnes raisons de supposer que ce que Kant a voulu dire n'a pas grand-chose à voir avec ce que l'adage signifie habituellement ${ }^{6}$. C'est pourquoi j'ai préféré placer Kant à l'origine du principe de parcimonie normative seulement, en dépit du fait que c'est plutôt la méta-norme " devoir implique pouvoir » qui est mise à son crédit la plupart du temps.

4. Kant, Emmanuel, Critique de la raison pratique, 1788, trad. Ferry, Luc et Wisman, Heinz, Paris, Gallimard, 1985, p. 62-63.

5. Von Wright, Georg Henrik, "Ought to be-Ought to do ", dans Six Essays in Philosophical Logic. Acta Philosophica Fennica, 60, 1996, p. 65.

6. Kant établit, semble-t-il, un lien d'équivalence entre conscience du devoir et conscience de la liberté. Cette idée peut être exprimée par l'affirmation "devoir implique pouvoir ", mais non par la formulation contraposée d'après laquelle s'il y a impossibilité de remplir l'obligation, l'obligation elle-même n'existe pas. Cf., Gardiès, Jean-Louis, L'erreur de Hume, Paris, PUF, 1987, p. 31. Voir aussi Gardiès, Jean-Louis, "Modalités et normes ", Archiv für Rechts und Sozialphilosophie, LXII, 4, 1976, 467. 
De toute façon, comme on pourra le constater très rapidement, l'examen qui suit n'a aucune visée exégétique. Ce n'est certainement pas une analyse fouillée des idées de Kant sur la question. Je souhaite seulement proposer quelques arguments en faveur d'une distinction entre deux modes de justification des méta-normes : le principe d'humanité et le principe de parcimonie, distinction qui est loin d'être évidente, en dépit du ton affirmatif que j'ai utilisé pour la présenter.

Pour finir, je soutiens que ces deux principes peuvent entrer en conflit.

D'un côté, le principe d'humanité nous demande de construire des théories morales qui ne sont pas trop exigeantes (c'est-à-dire d'exclure les normes cruelles ou inhumaines). D'un autre côté, le principe de parcimonie nous demande de construire de construire des théories morales suffisamment exigeantes (c'est-à-dire, d'exclure les normes superflues, celles, en particulier, qui s'alignent sur ce que l'agent veut déjà).

Il n'est pas du tout sûr qu'il soit possible de respecter ces deux demandes à la fois dans la construction de nos théories morales. C'est sur cette note pessimiste (pour les bâtisseurs de théories) que s'achève l'article.

\section{Normes et méta-normes}

Supposons qu'un agent de sécurité me dise : "Il est interdit de fumer dans l'enceinte du métro », et que je lui demande courageusement « Pourquoi ? ». Il ne serait pas très étonnant qu'il me réponde : "Parce que c'est la loi ». En posant la question, j'ai, pour ainsi dire, accompli un certain travail philosophique, puisque j'ai contribué à mettre en évidence une distinction, désormais classique (bien qu'exposée à différentes objections) entre l'énoncé normatif «Il est interdit de fumer dans l'enceinte du métro », qui est tel parce qu'il contient une expression dite " déontique " (c'est-à-dire de permission, d'interdiction, d'obligation) et la norme (règle, loi, prescription, directive, recommandation etc.) dont l'existence est, selon les théories, le «fondement », la « justification », la " condition de vérité ", le " sens », la " référence » etc. de l'énoncé normatif 7 .

À cette construction traditionnelle à deux niveaux, il me semble qu'il est possible d'en ajouter trois autres au moins. En effet, s'il est légitime de demander "En vertu de quoi est-il interdit de fumer dans le métro ? ", il ne l'est pas moins de poser la question : "Qu'est-ce qui vous autorise à dire que cette norme (loi, règle, etc.) existe ? ». Bref, il convient peut-être de présenter la norme invoquée à l'appui de tel ou tel énoncé normatif comme un simple " candidat à l'existence » en attente d'être sélectionné selon certains critères. L'un des critères les plus intéressants, me semble-t-il, est celui que Durkheim a fortement recommandé ${ }^{8}$.

7. Von Wright, Georg Henrik, "La fondation des normes et des énoncés normatifs ", Science, Technique, Société, 1986, p.10-26.

8. Durkheim, Emile, "Définition du fait moral» dans Textes 2 : Religion, morale, anomie, Paris, Minuit, 1975, p.257-288. 


\section{Philosophiques / Printemps 2001}

D'après lui, c'est l'existence de sanctions qui nous autorise à inférer l'existence de normes. Ce critère nous permet de distinguer assez clairement les normes juridiques des normes sociales ou morales. Les premières (juridiques) sont rendues manifestes par l'existence de sanctions dites " organisées ", c'est-à-dire administrées par des corps définis et constitués, selon une procédure connue d'avance. Ces sanctions (des amendes aux exécutions, en passant par l'emprisonnement) sont objectives, mesurables, observables. Les secondes (sociales et morales) s'expriment dans des sanctions dites "diffuses ", c'est-à-dire administrées par chacun et par tout le monde, sans procédure définie d'avance. Ces sanctions (sarcasmes, mépris, humiliation, etc.) et les peines associées (honte, perte de l'honneur, etc.) sont plutôt subjectives, difficiles à quantifier. Il est possible, bien sûr, de contester l'usage extensif qui est fait du terme « sanction » dans cette théorie. Il est possible, aussi, de proposer des critères d'identification de l'existence de normes un peu plus sympathiques. Il serait assez raisonnable de supposer qu'à la différence des normes juridiques, qui peuvent être des obligations ou des interdictions purement extérieures (comme les lois d'une puissance militaire occupante), les normes sociales ou morales doivent être approuvées, appréciées. L'existence d'une norme ne doit pas nécessairement être inférée de l'existence de sanctions : elle peut se manifester par son pouvoir d'attraction. ${ }^{9}$ Quoi qu'il en soit, tous ces critères sont situés au même niveau, celui auquel les normes juridiques sociales et morales peuvent être distinguées ${ }^{10}$. Il y a, cependant, des critères plus généraux qui permettent de sélectionner, dans l'ensemble des candidats à être des normes d'action ${ }^{11}$, qu'ils soient juridiques, sociaux, moraux, ceux qui peuvent être dits « authentiques » et de rejeter ceux qui recevront le nom peu flatteur de "pseudo-normes ${ }^{12}$. Le plus connu de ces critères se présente sous la forme d'une maxime disant « devoir implique pouvoir » ${ }^{13}$. Étant donné qu'il

9. C'est du moins ce que j'essaie de montrer dans Les causes et les raisons. Philosophie analytique et sciences humaines, Nîmes, J. Chambon, 1995, p.105-107.

10. Dans son essai non publié "Sens et normes ", que Maria-Michela Marzano-Parisoli a bien voulu me communiquer, on peut trouver une présentation très éclairante des différents critères qui servent habituellement à distinguer ces trois types de normes. Toutefois, l'article très complet et novateur d'Otto Pfersmann montre qu'il n'est vraiment pas facile de proposer des critères de distinction décisifs, ou plus exactement que les critères les plus courants ne sont pas décisifs, la tripartition elle-même méritant d'être remise en question. Cf. Pfersmann, Otto, "Pour une typologie modale de classes de validité normative ", dans, Petit, Jean-Louis, dir., La querelle des normes. Cahiers de philosophie politique et juridique, Presses Universitaires de Caen, 1995, p. 69-73.

11. À distinguer, par exemple, des normes épistémiques telles que "Acceptez les propositions vraies ; rejetez les propositions fausses ", pour lesquelles les principes de sélection ne semblent pas être identiques (c'est une question assez complexe que je préfère laisser de côté ici).

12. Von Wright Georg Henrik, "Y a-t-il une logique des normes ", dans Petit JeanLouis, dir., La querelle des normes., p. 36.

13. Dans la littérature de style analytique anglophone consacrée à cette question, cette méta-norme se présente sous la forme d'une relation entre termes (" ought » implies «can »), lorsque l'auteur veut insister leur lien logique. J'ai respecté cet usage. D’autre part, j'ai choisi 
s'applique à tous les candidats à être des normes, il n'est pas déraisonnable de l'appeler « méta-norme ».

Le rôle de ce critère dans la sélection des théories morales ne peut pas être négligé. Cette méta-norme sert, entre autres, à justifier la préférence pour certaines variétés de naturalisme moral ${ }^{14}$. Toutefois, parmi les philosophes qui exploitent la méta-norme pour justifier leur théorie, certains admettent qu'elle n'est pas une sorte de premier principe qui n'exige pas de justification (parce qu'elle serait évidente, etc.) Bref, la question "Pourquoi dites-vous "devoir implique pouvoir" ? " ne leur paraît pas moins légitime que " Pourquoi ditesvous qu'il est interdit de fumer dans le métro ? ». Enfin, "devoir implique pouvoir » n'est pas la seule méta-norme qui puisse jouer un rôle dans la sélection des théories morales. La méta-norme que j'ai attribuée à Kant, selon laquelle, il serait absurde de nous obliger à chercher à obtenir ce que nous voulons de toute façon, est dirigée contre les morales du bonheur personnel ${ }^{15}$.

$\mathrm{Au}$ total, nous avons donc cinq niveaux :1) l'énoncé normatif ; 2) l'affirmation de l'existence d'une norme ; 3) la justification de l'existence de normes spécifiques (juridiques, sociales, morales, etc.) ; 4) la sélection de l'ensemble des normes spécifiques au moyen de méta-normes ; 5) la justification des méta-normes.

Bien entendu, cette image des " niveaux » est assez dangereuse car elle risque de susciter l'objection de régression infinie. Il serait probablement plus prudent de se contenter d'opposer, comme on le fait habituellement, d'une

" devoir » implique " pouvoir ", de préférence à « devoir " suppose " pouvoir ", ou " devoir, c'est pouvoir ", parce que ma cible est précisément la maxime lorsqu'elle se présente comme un lien « logique » ou « conceptuel ».

14. Railton, "Made in the Shade: Moral Compatibilism and the Aims of Moral Theory », p. 105-106.

15. Cf., Kant, Critique de la raison pratique, p. 58-66. Il ne serait peut-être pas impossible de montrer que la méta-norme que Kant dirige (entre autres arguments, bien sûr), contre les morales du bonheur personnel, pourrait se retourner contre la théorie de Kant elle-même. Elle pourrait permettre d'établir, par exemple, qu'il n'existe pas de devoir de ne pas mentir, à proprement parler. Admettons, en effet, que le devoir de ne pas mentir puisse être justifié, en tant que devoir moral, par le fait que si nous voulions faire d'une maxime qui nous autorise à mentir un principe universel, nous serions inévitablement amenés à une contradiction (conceptuelle, pratique ou téléologique, selon les interprétations : Sullivan, Roger. J., Immanuel Kant's Moral Theory, Cambridge, Cambridge University Press, 1989, p.167-179). Un commandement ordonnant à chacun de ne pas mentir reviendrait, en somme, à un commandement de ne pas se contredire. Mais quelle serait l'utilité d'un tel commandement ? Nous cherchons "inévitablement " de nous-même à éviter de nous contredire. Un commandement de ne pas mentir, dans la mesure où il signifierait " ne pas se contredire ", serait donc " une sottise " au même titre que le commandement tourné en dérision par Kant, de chercher à se rendre heureux. En développant cet argument, on pourrait, je crois, aller jusqu'à se demander si cette méta-norme ne pourrait pas servir à exclure, de façon plus générale, les théories qui essaient de justifier l'autorité de certains principes moraux en invoquant leur rationalité (quelle que soit la conception de la rationalité endossée d'ailleurs). Mais, dans l'état rustique où se trouve encore cet argument, il vaut peut-être mieux ne pas chercher à aller aussi loin. 


\section{Philosophiques / Printemps 2001}

part l'énoncé normatif et, d'autre part, la norme, dont l'existence serait garantie par un ensemble de critères qui seraient considérés comme étant tous de même niveau (les méta-normes y compris). Mais j'insiste sur le fait qu'il ne s'agit que d'une image, que j'adopte sans m'engager sur les problèmes de régression parce qu'elle me permet de préciser que c'est à deux aspects seulement du problème des normes que je m'intéresse : les méta-normes qui s'appliquent à toutes les normes d'action et leur justification.

Par ailleurs, je tiens à souligner, que mon but n'est pas d'examiner dans tous ses détails la méta-norme "devoir implique pouvoir ", à propos de laquelle une littérature assez importante existe déjà ${ }^{16}$. Je ne commence pas, comme on a pris l'habitude de le faire, par recenser et discuter les différents sens de "devoir » (prescription, recommandation, nécessité pratique, attente, prédiction etc.) et de "pouvoir»(capacité, liberté, etc.). J'emploie « devoir » au sens le plus courant dans la discussion de la méta-norme qui m'intéresse, c'est-à-dire d'obligation et "pouvoir » au sens de possibilités logiques, physiques ou psychologiques. En d'autres mots plus compliqués, je pourrais dire que j'interprète cette méta-norme comme mettant en relation la modalité déontique de l'obligation et la modalité aléthique de la possibilité.

Ce que je souhaite montrer, seulement (si je puis dire), c'est que :

1) «devoir implique pouvoir » n'est pas la seule méta-norme qui puisse servir de critère de sélection des théories morales. La métanorme disant "Il est inutile d'ordonner à quelqu'un ce qu'il veut » (et la méta-norme dérivée «Il est inutile d'interdire à quelqu'un ce qu'il ne veut pas ») en est une autre.

2) si la méta-norme " devoir implique pouvoir » paraît inattaquable, ce n'est pas en raison d'une relation de dépendance conceptuelle entre «devoir » et pouvoir ", mais parce qu'elle repose sur un principe d'humanité auquel nous ne sommes pas prêts à renoncer.

3) la méta-norme disant qu'il est absurde d'ordonner à quelqu'un ce qu'il veut ou d'interdire à quelqu'un ce qu'il ne veut pas repose sur un tout autre principe, que j'appelle " principe de parcimonie ».

4) ces deux principes peuvent entrer en conflit.

16. Hare, Richard, M. " "Ought" and "Can" ", dans Freedom and Reason, Oxford, Clarendon Press, 1963, p. 51-66 ; Von Wright, Georg Henrik, Norm and Action, Londres, Routledge et Kegan Paul, 1963 ; Mavrodes, George I., " "Is" and "Ought" ", Analysis, XXV,2, 1964, p. 42-44 ; Shaw, P. D., "Ought and Can ", Analysis, XXV, 4, 1965, p.196-197; Hintikka, Jaako, "Deontic Logic : the Philosophical Morals ", Models for Modalities, Dordrecht, Reidel, 1969 ; Tranøy, " "Ought” implies "Can” : A Bridge from Fact to Norm "; Vermazen, Bruce, "The Logic of Practical "Ought" Sentences ", Philosophical Studies, 32, 1977, p.1-71 ; Brown, " Moral Theory and the Ought-Can Principle»; Thomas, Geoffrey, An Introduction to Ethics, Indianapolis, Hackett, 1993,p. 160-162 ; Yaffe, G., " "Ought” implies "Can" and the Principle of Alternate Possibilities ", Analysis, 59, 3, 1999, p.218-222 ; Carlson, "Oughts and Cans of Objective Consequentialism », Utilitas, 11, 1, 1999; Griffin, James, Value Judgments, Clarendon Press, Oxford, 1996, p. 89-92. À quoi on peut ajouter : Gardiès, Jean-Louis, L'erreur de Hume, Paris, PUF, 1987. 
L'idée qu'il n'y a pas de relation de dépendance conceptuelle entre " devoir » et " pouvoir » est au centre de mon argument. Je vais, bien sûr, commencer par essayer de donner des raisons en sa faveur.

\section{Le principe d'humanité}

Il existe différentes objections à l'idée qu'il existerait une sorte de relation conceptuelle entre " devoir » et " pouvoir ». La plus simple pourrait s'inspirer d'une remarque de Michael Stocker. ${ }^{17}$ Il n'est pas contradictoire de penser qu'un débiteur a le devoir de payer ses dettes, même s'il n'en a pas le pouvoir. Ce qui revient à dire qu'il n'y a pas de relation de dépendance conceptuelle entre « devoir » et " pouvoir».

Cependant, parmi les raisons qui devraient nous convaincre que la relation entre " devoir » et " pouvoir » n'est pas conceptuelle, certaines sont un peu plus argumentées. Les trois principales sont les suivantes :

1) « devoir implique pouvoir» contredit la loi de Hume.

2) " devoir implique pouvoir » exclut la possibilité de conflits moraux.

3) « devoir implique pouvoir » exclut la possibilité de la faiblesse de la volonté.

1) «Devoir implique pouvoir » contredit la loi de Hume.

La méta-norme « devoir implique pouvoir » peut être spécifiée de la façon suivante. «L'agent $\mathrm{X}$ ne doit faire $\mathrm{p}$ que s'il peut faire $\mathrm{p}$ ». Cette formulation est en accord avec certaines de nos intuitions relatives à la responsabilité. On ne blâme pas l'agent qui ne pourrait en aucun cas faire ce qu'il était censé devoir faire (à première vue). Mais la version contraposée de cette formulation semble poser un problème à tous ceux qui admettent ce qu'on appelle la "loi de Hume ". Selon cette loi, une conclusion normative ne peut pas être tirée de prémisses non normatives ${ }^{18}$. Or, la formule contraposée "Si l'agent $\mathrm{X}$ ne peut pas faire $\mathrm{p}$, alors il ne doit pas faire $\mathrm{p}$ » viole manifestement la loi de Hume puisqu'une conclusion normative (l'agent ne doit pas) est dérivée d'une prémisse non normative (l'agent ne peut pas) ${ }^{19}$. Il existe deux façons d'essayer d'écarter l'objection ${ }^{20}$ :

1) Nier que la conclusion soit normative en soutenant, par exemple, que la négation d'une norme n'est pas une norme.

17. Stocker, Michael, Plural and Conflicting Values, Oxford, Clarendon Press, 1990, p. 96.

18. Thomas, An Introduction to Ethics, p. 160-162 .

19. J'ai choisi " non normatif » de préférence à « descriptif » ou "factuel » car, comme Mavrodes le fait justement remarquer, opposer le normatif au factuel contient le présupposé (que je ne partage pas) selon lequel les jugements normatifs ne sont pas factuels. Cf, Mavrodes, " "Is" and "Ought" ", p.42, note 2.

20. Thomas, An Introduction to Ethics, p.161-162. 
2) Nier que les prémisses soient non normatives en soutenant, par exemple, que «Si on ne peut pas, on ne doit pas » est un enthymème, un raisonnement dont l'une des prémisses n'a pas été formulée explicitement. Le raisonnement complet aurait l'allure suivante :

Prémisse : Si l'agent X ne peut pas faire $\mathrm{p}$, il ne doit pas faire $\mathrm{p}$.

Prémisse : l'agent $\mathrm{X}$ ne peut pas faire $\mathrm{p}$.

Conclusion : l'agent $\mathrm{X}$ ne doit pas faire $\mathrm{p}$.

Dans ce raisonnement, la prémisse majeure est normative, de sorte qu'aucune conclusion normative n'est tirée de prémisses purement non normatives.

Ni l'une ni l'autre de ces manœuvres n'est tout à fait convaincante.

L'idée que la conclusion est non normative est parfois justifiée par une interprétation pragmatique de "devoir implique pouvoir ». En fait, il ne faudrait pas dire " devoir » implique " pouvoir ", mais " devoir » présuppose " pouvoir ». Et, de même que l'attribution de propriétés physiques à une chose présuppose son existence, de même l'attribution de propriétés déontiques à une action (permise, obligatoire, interdite) présuppose que cette action soit possible. S'il est clair que l'action ne peut pas être accomplie, la question ne se pose pas de savoir si elle est permise, obligatoire, interdite : il ne peut tout simplement pas y avoir de norme relative à cette action ${ }^{21}$. Si on rejette cette interprétation pragmatique, on se retrouve avec une difficulté logique. Selon George Mavrodes, l'idée que la négation d'une norme pourrait ne pas être une norme laisse penser qu'il pourrait y avoir des énoncés normatifs dont la négation serait non normative, ce qui est plutôt bizarre ${ }^{22}$. Pourquoi «bizarre »? Supposons qu'on me demande de juger si X doit faire p. Si la négation d'une norme n'est pas une norme, il s'ensuivra que si je dis "X doit faire $\mathrm{p}$ », mon jugement sera normatif, et si je dis « X ne doit pas faire $\mathrm{p}$ », mon jugement ne sera pas normatif. C'est, en effet, bizarre. D'autre part, il faudrait pouvoir résoudre le cas complexe de la double négation. À supposer que la négation d'une norme ne soit pas une norme, qu'en est-il de la négation de cette négation ? Est-elle une norme ou pas ? Si on estime qu'elle est une norme, comme il semble naturel de le faire, on se retrouve avec la difficulté soulignée par Geoffrey Thomas $^{23}$. Il serait étrange que la négation d'une norme ne soit pas une norme alors que la double négation d'une norme est une norme. Ceux qui tiennent à l'idée que la conclusion « $\mathrm{X}$ ne doit pas » n'est pas normative ont le recours, semble-t-il de distinguer deux formes de négation. La négation de portée étroite de « $\mathrm{X}$ doit faire $\mathrm{p}$ » serait la norme : "X ne doit pas faire $\mathrm{p}$ ». Mais la négation de portée large de « X doit faire $\mathrm{p}$ », c'est-à-dire « Il n'est pas vrai que X doit faire p », signifierait «Il n'y a pas de norme relative à $\mathrm{p}$ ". Ils diront ensuite que, de même

21. Shaw, "Ough and Can ", p.196-197.

22. Mavrodes. "Is" and "Ought" ", p. 43.

23. Thomas, An Introduction to Ethics, p.162 
que la négation large d'une norme n'est pas une norme mais le constat de l'absence de toute norme, la négation de cette négation n'est pas une norme, mais le constat de l'existence d'une norme quelle qu'elle soit. Serons-nous débarrassés de la difficulté pour autant ? C'est peu probable, car on ne voit pas très bien pourquoi une conclusion normative non spécifiée devrait être jugée non normative.

La seconde manœuvre est également exposée à différentes objections. La principale est la suivante. La formulation contraposée de " devoir » implique "pouvoir ", est présentée comme un raisonnement dont la prémisse cachée serait la norme "Si X ne peut pas faire $p, X$ ne doit pas faire p ». L'avantage, c'est que la loi de Hume est respectée, puisque le raisonnement va du normatif au normatif. L'inconvénient, selon Mavrodes, c'est que " devoir » implique " pouvoir » n'est plus considéré comme une vérité logique ou conceptuelle. La méta-norme devient un principe du même genre que celui qui nous interdit de tuer des innocents ${ }^{24}$. Il n'y a, en effet, aucune bonne raison de juger que "Si X ne peut pas faire $p, X$ ne doit pas faire $p$ » est une norme et que «Si X doit faire $\mathrm{p}, \mathrm{X}$ peut faire $\mathrm{p}$ » n'en est pas une.

D’un côté, la méta-norme " devoir implique pouvoir » dans sa version contraposée ( "Si on ne peut pas, on ne doit pas ») est invoquée pour justifier une certaine forme de scepticisme à l'égard de la loi de Hume, c'est-à-dire de l'idée qu'il serait impossible de tirer des conclusions normatives de prémisses non normatives. D’un autre côté, la loi de Hume est invoquée pour contester le caractère inattaquable de "devoir implique pouvoir ». Dans sa version contraposée, la méta-norme ne respecte pas la loi de Hume, et doit, pour cette raison, être rejetée. Mais, en réalité, tout ce que les partisans de cette dernière thèse seraient susceptibles d'avoir montré, c'est qu'il existe des raisons de rejeter la méta-norme "devoir implique pouvoir ", lorsqu'elle est présentée comme une sorte de vérité conceptuelle.

\section{2) «Devoir implique pouvoir » exclut la possibilité de conflits moraux}

D'après Bernard Williams, un conflit (ou un dilemme) moral peut prendre deux formes fondamentales ${ }^{25}$ :

1) Je dois faire a et je dois faire b, mais je ne peux pas faire a et b

2) Je dois faire $c$ et ne pas faire $c$

On peut illustrer la première forme de la façon suivante ${ }^{26}$. Supposons qu'après une délibération ultra rapide, j'arrive à la conclusion que je dois sauter à l'eau pour essayer de sauver un enfant qui est en train de se noyer à ma droite dans un lac. Admettons que le jumeau de cet enfant soit en train de se

24. Mavrodes, "Is" and "Ought" ", p.44.

25. Williams, "La cohérence éthique », p. 115.

26. Exemple inspiré de Barcan Marcus, Ruth, "Moral Dilemmas and Consistency ", Journal of Philosophy, LXXVII, 3, 1980, p.121-136. 


\section{8 · Philosophiques / Printemps 2001}

noyer dans le même lac à ma gauche mais à une distance telle que je ne puisse pas sauver les deux jumeaux à la fois. En vertu d'un principe d'impartialité, qui nous demande de traiter les cas similaires de façon similaire, s'il est vrai que je dois essayer de sauver l'un, il est vrai aussi que je dois essayer de sauver l'autre. Autrement dit, je dois sauver les deux. Mais c'est, évidemment, une chose que je ne peux pas faire. Ce genre de cas nous permet d'envisager la possibilité que « devoir » n’implique pas nécessairement « pouvoir».

Supposons, à présent, que la personne qui se noie n'est pas un enfant innocent mais un individu maléfique ayant détruit une partie de l'humanité et qui, je le sais, a manifesté l'intention d'en détruire une autre. Pour certains philosophes, il ne serait pas absurde de dire que, dans des cas de ce genre, je dois la sauver et je ne dois pas la sauver, ce que je ne peux pas faire ${ }^{27}$. Ces cas illustrent la deuxième forme de conflit moral. Ils semblent suggérer, eux aussi, que « devoir » n'implique pas nécessairement " pouvoir ».

L'objection qui vient assez naturellement à l'esprit, c'est que, dans les deux cas, c'est une erreur de croire qu'un devoir existe ou que le devoir soit bien celui qui est décrit. Il est permis de penser que des devoirs contradictoires (faire $\mathrm{c}$ et ne pas faire $\mathrm{c}$ ) ne sont pas des devoirs du tout ${ }^{28}$. D'autre part, il est tout fait raisonnable de considérer que le devoir, lorsqu'il y en a deux d'égale importance et qu'il est impossible de les respecter simultanément, n'est pas de faire les deux, mais l'un ou l'autre ${ }^{29}$. De ceci il semble suivre que "devoir » implique " pouvoir " n'est pas menacé par les cas en question, puisque, dans le premier, il n'y a pas de devoir à proprement parler et dans le second, un " devoir » auquel correspond un " pouvoir ». Mais E. J. Lemmon est d'un avis différent. Il estime que « Je dois » et « Je ne dois pas " ne sont pas contradictoires, à proprement parler : les deux peuvent être vrais simultanément. Et, d'après lui, admettre la possibilité du conflit moral, c'est, tout simplement, reconnaître qu'un devoir et sa négation peuvent être vrais simultanément. Qu'il nous arrive d'être obligés de faire et de ne pas faire quelque chose est un " fait désolant propre à la vie humaine, mais ce n'est pas une contradiction $\operatorname{logique}^{30} »$. On lui a fait remarquer, toutefois, que, même si pris en eux-

27. L'exemple de Lemmon, que j'ai adapté, lui-même adapté de Platon, est approximativement le suivant. Un ami vous laisse un fusil de chasse en dépôt. Vous promettez de le lui rendre dès qu'il repassera le chercher. Mais lorsque cet ami revient pour récupérer son arme, il est dans un état de totale dépression et vous craignez qu'il s'en serve pour autre chose que du gibier (sa femme, ses enfants, lui-même, etc.). Dans un cas de ce genre, dit Lemmon, on doit et on ne doit pas restituer le fusil. Lemmon, E. J., " Moral Dilemmas », Philosophical Review, LXXI, 2, 1962, p.150.

28. Conee, Earl, "Why Moral Dilemmas are Impossible ", American Philosophical Quarterly, 26, 1989, p.133-141.

29. Sur les problèmes que pose cette solution dite "disjonctive ", et sur toutes sortes d'autres difficultés liées aux conflits ou aux dilemmes moraux, voir Tappolet, Christine, "Dilemmes moraux », dans Canto-Sperber, Monique, dir., Dictionnaire d'éthique et de philosophie morale, 2ème éd., Paris , PUF, 1996

30. Lemmon, "Moral Dilemmas », p. 150. 
mêmes, un énoncé d'obligation et sa négation ne sont pas contradictoires, ils le deviennent si on introduit "devoir " implique "pouvoir ${ }^{31}$. En effet, si « devoir » implique " pouvoir », de « Je dois » et "Je ne dois pas », qui ne sont pas contradictoires, il suit « Je peux » et "Je ne peux pas ", qui sont contradictoires $^{32}$. Confronté à cette difficulté, Lemmon conclut qu'il est préférable de renoncer à " devoir » implique " pouvoir » plutôt qu'à l'idée qu'un devoir et sa négation peuvent être vrais simultanément (c'est-à-dire au conflit moral tel qu'il le conçoit). Cette conclusion met clairement en évidence l'alternative qui m'intéresse. Ou bien on tient à l'idée de conflit moral, mais, dans ce cas, il faut renoncer à " devoir " implique "pouvoir ". Ou bien, on tient à " devoir » implique " pouvoir », mais dans ce cas il faut renoncer à l'idée de conflit moral. Ceux qui n'ont pas le goût des solutions extrêmes et veulent échapper à cette alternative qu'ils jugent peu séduisante ont le recours, semble$\mathrm{t}$-il de tenter de montrer que les conflits que Lemmon envisage, de la forme « Je dois faire c et ne pas faire c ", peuvent être ramenés à des conflits de la forme " Je dois faire $a$ » et " Je dois faire $b{ }^{33}$. Il ne leur reste plus ensuite qu'à proposer une solution de type disjonctif (ou bien a ou bien b) qui n'est pas incompatible avec «devoir » implique "pouvoir ». Mais les solutions de type disjonctif sont plutôt celles qui nient l'existence de conflits moraux. Elles laissent donc, me semble-t-il, l'alternative en l'état . Ce qui revient à dire que Lemmon n'a probablement pas tort. On pourrait toutefois lui reprocher de jeter le bébé avec l'eau du bain. Mais le bébé peut être sauvé. À supposer, en effet, qu'il $\mathrm{y}$ ait des raisons de renoncer à " devoir implique pouvoir » dans sa version conceptuelle ou logique, il ne s'ensuivrait pas qu'il y aurait des raisons de la rejeter dans toutes ses versions.

3) « devoir implique pouvoir » exclut la possibilité de la faiblesse de la volonté

L'argument dit de « la faiblesse de la volonté » contient l'idée qu'il est parfaitement concevable qu'un devoir soit reconnu comme tel alors qu'il est psychologiquement impossible de le respecter ${ }^{34}$. Il y a toutes sortes de cas dans lesquels nous avons l'impression que nous ne faisons pas ce que nous pen-

31. Ibid., note 8, p.150.

32. Ce qu'il est permis de contester. Jon Elster me propose l'objection suivante. De « Je dois » et «Je ne dois pas ", il ne suit pas «Je peux » et « Je ne peux pas », car même si de «Je dois » il suit « Je peux » (par « devoir implique « pouvoir»), de « Je ne dois pas », il ne suit pas "Je ne peux pas ». Je comprends l'objection ainsi : il est évident que, parmi les choses que je ne dois pas faire, il y en a que je peux faire : «Je ne dois pas » n'implique pas « Je ne peux pas ». Personnellement, ce que je tire de l'objection, c'est que le terme «implique » dans la maxime «devoir implique pouvoir» ne doit pas être pris au sens fort ou logique, en raison de l'asymétrie entre " Je dois » et « Je peux » d'une part, " Je ne dois pas » et « Je ne peux pas », de l'autre.

33. À la suite de Bernard Williams, "La cohérence éthique ", p.117.

34. Vermazen, «The Logic of Practical «Ought» Sentences », p. 62-63. 
sons devoir faire à cause d'une défaillance insurmontable de notre volonté (passagère ou permanente, c'est sans importance dans cette discussion). Pourtant, nous n'en tirons pas nécessairement la conclusion que le devoir n'était pas authentique. Ce qui revient à dire qu'il y a un cas, au moins, où « devoir " n'implique pas " pouvoir " : le cas où " pouvoir » est pris au sens psychologique d' "avoir la volonté ". Mais il est permis de soutenir que "devoir " n'implique pas " pouvoir " au sens psychologique. Un devoir auquel nous ne pourrions pas obéir en raison d'une défaillance insurmontable de notre volonté resterait un devoir. "Devoir » impliquerait seulement "pouvoir » au sens logique (un devoir qu'il serait impossible de respecter parce qu'il est contradictoire serait inauthentique) et au sens physique (un devoir qu'il serait impossible de respecter car il viole les lois de la nature serait inauthentique). Bref, si les impossibilités psychologiques ne sont pas prises en ligne de compte, le principe est à l'abri des objections de type "faiblesse de la volonté », mais on peut avoir des raisons de le trouver trop pauvre dans le contexte d'une discussion relative aux normes d'action. Si les impossibilités psychologiques de type "faiblesse de la volonté » sont prises en ligne de compte, le principe est intéressant, mais il est attaquable. On trouvera toujours des philosophes pour dire qu'une obligation peut être valide même si nous n'avons pas la possibilité psychologique de la respecter. Finalement, la discussion du problème dit de la "faiblesse de la volonté » nous confronte à une alternative similaire aux précédentes. Ou bien nous admettons « devoir implique pouvoir » et la possibilité de la faiblesse de la volonté, c'est-à-dire de cas dans lesquels on doit mais on ne peut pas au sens psychologique (défaillance insurmontable de la volonté) est exclue. Ou bien nous admettons la possibilité de la faiblesse de la volonté et nous devons renoncer à " devoir implique pouvoir » au sens fort de la formule, c'est-à-dire d'une relation dont la négation serait inconcevable.

L'ensemble des arguments dirigés contre « devoir implique pouvoir » semble bien montrer qu'il n'y a pas de lien interne ou conceptuel entre « devoir » et " pouvoir ». On peut très bien concevoir des devoirs auxquels il est impossible de se conformer pour des raisons psychologiques ou parce qu'ils sont en conflit avec d'autres devoirs, mais qui restent, néanmoins, des devoirs authentiques. D'autre part, on peut penser qu'il convient de rejeter " devoir implique pouvoir » dans sa version conceptuelle parce qu'on estime qu'elle est incompatible avec la loi de Hume.

À la suite de Tranøy, il ne paraît pas illégitime de conclure que, dans notre monde au moins, il n'est pas logiquement impossible d'exiger l'impossible ${ }^{35}$. Mais, à supposer qu'il serait en effet, logiquement possible d'exiger l'impossible, il n'en résulterait pas que nous devrions le faire. À quoi pourrait bien ressembler un monde où l'impossible serait exigé ? À un monde bizarre (pour dire le moins), cruel et inhumain (pour dire ce qu'il

35. Tranøy «'Ought' Implies ‘Can' : A Bridge from Fact to Norm », p.122. 
serait assez exactement, puisque ces normes seraient, en principe, assorties de sanctions négatives et donc de souffrances injustifiées).

À supposer, donc, que la méta-norme « devoir implique pouvoir » n’ait pas de soutien logique, elle resterait justifiée, néanmoins, par ce que j'appelle un principe d'bumanité. "Devoir implique pouvoir » est une méta-norme qui nous enjoint d'exclure les candidats à être des normes, qui, s'ils étaient retenus, rendraient notre monde cruel ou inhumain ${ }^{36}$. Si nous jugeons que "devoir implique pouvoir " est inattaquable, c'est, à mon avis, pour cette raison. En tout cas, cela ne peut pas être pour des raisons logiques, car de ce point de vue, la méta-norme est loin d'être intouchable.

D'après Tranøy, de «Si une action p est impossible, il n'est pas permis qu'elle soit obligatoire ", on peut dériver "Si une action p est nécessaire, il n'est pas permis qu'elle soit interdite ${ }^{37}$. Autrement dit, de la méta-norme qui exclut d'obliger l'impossible, on peut dériver la méta-norme qui exclut d'interdire le nécessaire. J'ajouterai que ces deux méta-normes sont justifiées par le principe d'humanité. Un monde dans lequel ce qui est nécessaire (à différents points de vue : physique, psychologique, etc.) serait interdit, serait aussi cruel et inhumain qu'un monde où ce qui est impossible serait obligatoire.

Bien entendu, le principe d'humanité est plus général que la métanorme « devoir implique pouvoir ». Il nous enjoint de rejeter toutes les obligations cruelles, celles que l'agent ne peut pas remplir n'étant qu'un cas particulier. Certains philosophes estiment que les devoirs d'impartialité sont cruels, mais ils pourraient très bien admettre qu'il n'est pas impossible d'y obéir. Ils diraient seulement qu'il ne faut pas toujours agir conformément à ce qu'ils exigent. Ils pourraient ajouter : " en vertu du principe d'humanité ». Mais ce qui m'intéresse ici, ce sont les cas dans lesquels les obligations sont cruelles précisément parce qu'on ne peut pas les honorer. Par ailleurs, il n'est peut-être pas inutile d'insister sur le fait que, ce qui est cruel, ce n'est pas de formuler une obligation de faire l'impossible, mais de sanctionner ceux qui violent l'obligation ${ }^{38}$. Imaginons une situation ubuesque (proche de notre actualité, en fait) dans laquelle l'obtention d'une carte de travail serait conditionnée par l'obtention d'une carte de séjour et l'obtention d'une carte de séjour par celle d'une carte de travail. Une norme exigeant l'impossible dans ces conditions (posséder une carte de séjour) serait seulement grotesque, si

36. Ibid., p.123.

37. Ibid., p.121.

38. J'ai introduit cette précision à la suite d'un commentaire d'Otto Pfersmann (merci). Cependant, j'ai choisi de ne pas le suivre jusqu'au bout de son analyse (pour le moment). Si je l'ai bien compris, une norme qui exige l'impossible peut masquer la volonté de maltraiter systématiquement certaines personnes, volonté qui s'exprime effectivement dans des normes qui autorisent des actes de violence à l'égard de ces personnes. Mais, en tant que telle, la norme qui exige l'impossible est bel et bien dépourvue de sens, ce que je conteste d'une certaine façon puisque j'estime qu'il n'y a pas de relation conceptuelle ou logique entre "devoir » et « pouvoir ». Ce que je propose ici est une tentative de compromis. 
l'infraction à cette norme n'était pas sanctionnée par l'expulsion (ou pire, selon les cas et l'imagination du législateur).

\section{Le principe de parcimonie}

Revenons au «rasoir de Kant ». Ce rasoir élimine toutes les normes qui ordonnent à quelqu'un ce qu'il veut inévitablement de lui-même. Étant donné que cette méta-norme est relative à la conduite humaine, cette formule intentionnelle (en ce sens qu'elle fait référence au " vouloir » de l'agent) est préférable à d'autres qui seraient purement comportementales (telles que : " ce que l'agent ferait de toute façon »). De ce point de vue, la formulation kantienne est adéquate. D'un autre côté, il me semble qu'elle pourrait être débarrassée de la spécification modale : l'adverbe «inévitablement " pourrait être éliminé. On pourrait dire simplement qu'en vertu de certaines considérations pragmatiques, il est absurde d'ordonner à quelqu'un de faire ce qu'il veut de lui-même (qu'il le veuille « inévitablement » ou pas). Un ordre commandant à quelqu'un de faire ce qu'il veut de lui-même serait aussi absurde qu'un ordre commandant à quelqu'un de fermer la porte alors qu'elle est fermée ou de l'ouvrir alors qu'elle est ouverte.

En réalité, il existe tout un ensemble de conditions qui, si elles ne sont pas satisfaites, privent l'ordre de sa validité, si on peut dire. Il reste un ordre du point de vue sémantique, mais il n'est pas un ordre du point de vue pragmatique. Parmi ces conditions, il y a très certainement celles que John Searle a mises en évidence. Un ordre d'accomplir une action est défectueux si la personne à qui il s'adresse est déjà en train de l'accomplir ou si elle s'apprête à l'accomplir tout à fait indépendamment de l'ordre ${ }^{39}$. Bref, un ordre d'accomplir une action est sans objet si la personne à qui il s'adresse voulait ou désirait accomplir cette action.

Frankena avait proposé de traiter la méta-norme « devoir implique pouvoir » de façon pragmatique :

« devoir implique pouvoir» ne doit pas être nécessairement interprété comme une implication logique stricte. On peut soutenir, de façon plausible, que c'est une affirmation disant : a. les jugements moraux «présupposent » ou « impliquent pragmatiquement » que l'agent puisse accomplir l'action...; b. l'objet du jugement moral disparait si les agents ne peuvent pas accomplir l'acte ; c. il serait moralement injuste d'insister sur le fait qu'un agent doit accomplir une action certaine action, s'il est supposé ne pas pouvoir l'accomplir ${ }^{40}$.

C'est la troisième interprétation que j'endosse ici (il serait moralement injuste...). Tout ce que je voudrais ajouter, c'est qu'elle n'est pas plausible

39. Searle, John, Les actes de langage. Essai de philosophie du langage (1969), trad. H. Pauchard, Paris, Hermann, 1972, p. 101.

40. Frankena, William, "Obligation and Motivation in Recent Moral Philosophy ", dans Melden, A. I., ed., Essays in Moral Philosophy, Seattle, University of Washington Press, 1954, p. 60 
pour la méta-norme qui nous demande d'exclure les normes qui nous obligent à faire ce que nous voulons de nous-mêmes. En quoi le fait d'obliger quelqu'un à faire ce qu'il veut serait-il moralement injuste ? Un monde dans lequel nous serions obligés de faire ce que nous voulons ne serait pas cruel ou inhumain. Il serait seulement encombré de normes sans objet qu'un principe d'économie ou de parcimonie devrait, en principe, éliminer ${ }^{41}$

\section{Deux méta-normes justifiées par le principe de parcimonie : « Il est inutile d'ordonner à quelqu'un ce qu'il veut », « Il est inutile d'interdire à quelqu'un ce qu'il ne veut pas »}

La méta-norme qui exclut les candidats à être des normes qui nous demandent de faire ce que nous voulons est-elle la seule que le principe de parcimonie puisse justifier ? Non. En vertu des mêmes considérations pragmatiques, il existe une méta-norme qui exclut les normes qui interdisent de faire ce que nous ne voulons pas. Claude Levi-Strauss fait implicitement appel à cette méta-norme pour ridiculiser certaines théories destinées à expliquer la prohibition de l'inceste. Selon ces théories, si l'inceste fait l'objet d'une prohibition universelle, c'est parce que les membres de l'espèce humaine ont une répulsion naturelle à l'égard des relations sexuelles avec les parents les plus proches. Mais, demande pertinemment Lévi-Strauss, s'il existait une répulsion naturelle à l'égard des relations sexuelles avec les parents les plus proches, à quoi servirait la règle qui l'interdit solen-nellement ? D'après lui, "Il n'existe aucune raison de défendre ce qui, sans défense, ne risquerait point d'être exécuté $^{42} »$. L'idée sous-jacente, bien sûr, c'est qu'il est superflu, inutile, etc.

41. Je me demande, néanmoins, si des problèmes liés aux conditions de satisfaction des ordres ne risquent pas de rendre les choses un peu moins tranchées. Même si le donneur d'ordre ne fait que devancer, pour ainsi dire, le vouloir du récepteur, on peut estimer qu'il agit de façon humiliante parce qu'il ne tient pas compte du vouloir de l'agent («Vous allez faire p, que vous le vouliez ou non!»). Jon Elster a proposé des analyses très excitantes de ces complications et d'autres, liées aux conditions de satisfaction des ordres dans Elster, Jon, Le laboureur et ses enfants, trad. A. Gerschenfeld, Paris, Minuit, 1986, p. 38-46. Il pourrait en ressortir que c'est le principe d'humanité et non le principe de parcimonie qui justifie la méta-norme qui nous demande de ne pas ordonner à quelqu'un ce qu'il veut. Je ne crois pas, toutefois, que ces complications pourraient remettre en question l'existence de deux ordres de justification des méta-normes (humanité et parcimonie). Elles laisseraient seulement supposer que, dans certains cas, ces deux principes peuvent s'appliquer sans entrer en conflit.

42. Levi-Strauss, Claude, Les structures élémentaires de la parenté, Paris, La Haye, Mouton, 1947, p.21. Levi-Strauss prend en considération deux objections à son argument. La prohibition " n'est destinée qu'à des cas exceptionnels où la nature faut à sa mission » (p. 21). À cela, il répond que si l'objet de la prohibition n'était que ces cas exceptionnels, il y aurait une disproportion inexplicable entre la modestie du phénomène à réprimer et l'importance de la réglementation qui le vise, la rigueur des sanctions qui punissent sa violation. D'autre part, le suicide semble être un contre exemple, puisqu'il arrive que la loi le combatte par des sanctions multiples « bien que la tendance à la préservation soit naturelle à l'être vivant»(p.21). À cela, Lévi-Strauss répond que si toute société interdit l'inceste, « il n'est aucune qui ne fasse sa place 
d'interdire ce que nous ne voulons pas. Cependant, si le raisonnement de LéviStrauss était inattaquable du début à la fin, il faudrait aussi accepter celui, similaire, de Voltaire, dont l'absurdité est manifeste, qui dit, en gros «Ce ne serait pas interdit dans la Bible si cela n'avait pas été pratiqué ${ }^{43} »$.

Selon Voltaire, l'invention du Décalogue ne peut pas être mise au bénéfice des juifs. Le fait qu'ils inventèrent, pour ainsi dire, les lois strictes prohibant le vol, le meurtre, le blasphème, la convoitise, témoigne tout simplement du fait que ces pratiques étaient extrêmement répandues parmi eux. Si tel n'était pas le cas, pourquoi auraient-ils promulgué ces lois ?

Ce qui ne va pas, dans l'argument de Lévi-Strauss et de Voltaire, c'est (entre autres) qu'ils ne tiennent pas compte du caractère normatif du principe de parcimonie. De l'existence de normes d'interdiction, ils concluent à l'existence des actions ou des inclinations sanctionnées par ces normes. Mais ce qu'ils sont autorisés à affirmer, seulement, c'est qu'il en irait ainsi si le principe de parcimonie était toujours respecté. Or, même s'il existe un principe qui nous demande d'éliminer les normes superflues, rien ne dit qu'il est toujours respecté. Il y a toutes sortes de normes qui ne sont pas suivies bien qu'elles soient officiellement en vigueur, ou toutes sortes de normes « superflues » dérivées de normes qui ne le sont pas, qu'il serait trop coûteux d'éliminer, etc. Par ailleurs, Voltaire et Lévi-Strauss semblent supposer que l'existence de normes d'interdiction prouve l'existence préalable des actions ou des inclinations sanctionnées par ces normes. Mais il est permis de supposer que ces actions ou ces inclinations n'existeraient pas en l'absence de ces normes. Après tout, saint Paul n'avait peut-être pas eu tort d'affirmer qu'il aurait ignoré la convoitise si la Loi n'avait dit : "Tu ne convoiteras pas ! " ${ }^{44}$. Bref, de l'existence de normes d'interdiction, on ne peut pas conclure à l'existence préalable des actions ou des inclinations sanctionnées par ces normes sans argument supplémentaire.

Tout cela ne remet pas en question l'existence des deux méta-normes justifiées par le principe de parcimonie normative, mais seulement l'exploitation incorrecte ou abusive qui peut en être faite. Ces méta-normes peuvent être formulées de la façon suivante :

- Il est inutile d'ordonner à quelqu'un ce qu'il veut.

- Il est inutile d'interdire à quelqu'un ce qu'il ne veut pas.

au suicide »(p.22), ce qui rend la comparaison problématique à différents points de vue. On ne peut pas dire que ces réponses anéantissent les objections, mais elles suffisent, me semble-t-il, à laisser supposer qu'elles ne ruinent pas nécessairement l'ensemble du raisonnement de Lévi-Strauss.

43. "On tire de la punition du coït avec les bêtes une preuve que les juifs étaient forts enclins à cette abomination » écrit Voltaire, note 1, p.181, dans La Bible enfin expliquée, O.C. éd. 1818. C'est Frédéric Nef qui a attiré mon attention sur cet aspect peu connu des raisonnements de Voltaire (dont on aimerait penser qu'ils ne sont qu'ironiques !). Nef, Frédéric, "Tolérance et résignation. Remarques sur l'antisémitisme de Voltaire ", dans Voltaire, Rousseau et la tolérance, Amsterdam, Maison Descartes, 1977, p.124-125.

44. Saint Paul, Epître aux Romains. Epître aux galates, Paris, Garnier-Flammarion, p. $98-99$. 
Mais on pourrait probablement trouver d'autres formulations équivalentes, en raison des possibilités d'interdéfinition des expressions modales.

\section{Comment les principes d'humanité et de parcimonie peuvent entrer en conflit}

La méta-norme « devoir implique pouvoir » n'exclut, a priori, aucun genre d'impossibilité. Elle peut admettre des impossibilités psychologiques, aussi difficiles à établir soient-elles (les cas de faiblesse de la volonté évoqués, par exemple). Il est donc permis de soutenir que, pour qu'une norme d'obligation soit authentique, son contenu doit faire partie de l'ensemble de ce que l'agent peut vouloir en vertu de la méta-norme disant " devoir implique pouvoir ». Il est aussi permis d'ajouter que, lorsque l'agent est arrivé au point où il veut de luimême ce que l'obligation exige, cette dernière devient superflue en vertu de la méta-norme disant qu'il est inutile d'ordonner à quelqu'un ce qu'il veut.

Le parcours de l'obligation authentique est donc limité à la distance allant de ce que l'agent peut vouloir à ce qu'il veut effectivement ${ }^{45}$. Tout cela peut être traduit dans un ensemble de formules indiquant l'existence d'un conflit potentiel entre les principes d'humanité et de parcimonie .

1) Si l'écart entre l'obligation et le vouloir est nul, l'obligation est exclue en vertu du principe de parcimonie.

2) Si l'écart entre l'obligation et le vouloir est trop important, l'obligation est exclue en vertu du principe d'humanité.

3) Si l'écart entre l'obligation et le vouloir n'est ni nul ni trop important, l'obligation est authentique (elle sert en fait à amener progressivement le vouloir à se conformer à l'obligation).

Mais il faut reconnaître qu'il n'est pas très facile de donner un sens stable à l'idée d'un écart ni nul ni trop important. D'où la possibilité de conflits relatifs à l'authenticité des devoirs, auxquels on ne pourrait apporter que des solutions essentiellement contestables ${ }^{46}$.

45. Il faudrait peut-être ajouter ici : dans la mesure où l'on endosse l'argument de Bernard Williams qui nie l'existence de raisons d'agir « externes ». En effet, pour un défenseur des raisons externes, le parcours de l'obligation authentique n'est pas limité par ce que l'agent peut vouloir. Cf. Williams, Bernard, "Internal and External Reasons » dans Moral Luck, 1981,p. 101-113, et l'excellente critique de Stéphane Lemaire, "Raisons internes, raisons, raisons réelles ", manuscrit non publié.

46. Ce texte est une version remaniée d'une communication présentée au colloque de la SOPHA, en juin 2000, puis au séminaire de philosophie morale du CREA, en février 2001. Merci à Monique Canto-Sperber, Otto Pfersmann, Christine Tappolet, Daniel Weinstock et deux évaluateurs anonymes de la revue pour leurs commentaires sur la première version, ainsi qu'à Jean-Pierre Dupuy, Jon Elster, Philippe Mongin, qui ont discuté la seconde avec énergie. Merci aussi à Dominique Berlioz, Richard Glauser, Pierre Livet, Stéphane Lemaire, MariaMichela Marzano-Parisoli, Frédéric Nef, Luca Parisoli, Peter Railton dont les remarques sur la question m'ont beaucoup aidé. 